\title{
Halo Wide Binaries and Moving Clusters as Probes of the Dynamical and Merger History of our Galaxy
}

\author{
Christine Allen $^{1}$, Arcadio Poveda ${ }^{1}$ and A. Hernández-Alcántara ${ }^{1}$ \\ ${ }^{1}$ Instituto de Astronomía, Universidad Nacional Autónoma de México, Ciudad Universitaria, \\ 04510 México D.F. \\ email: chris@astroscu.unam.mx
}

\begin{abstract}
Wide or fragile pairs are sensitive probes of the galactic potential, and they have been used to provide information about the galactic tidal field, the density of GMC and the masses of dark matter perturbers present in both the disk and the halo. Halo wide binaries and moving clusters, since they are likely to be the remains of past mergers or of dissolved clusters, can provide information on the dynamical and merger history of our Galaxy. Such remnants should continue to show similar motions over times of the order of their ages. We have looked for phase space groupings among the low-metallicity stars of Schuster et al. (2006) and have identified a number of candidate moving clusters. In several of the moving clusters we found a wide CPM binary already identified in our catalogue of wide binaries among highvelocity and metal-poor stars (Allen et al. 2000a). Spectroscopic follow-up studies of these stars would confirm the physical reality of the groups, as well as allow us to distinguish whether their progenitors are dissolved clusters or accreted extragalactic systems.
\end{abstract}

Keywords. Galaxy: halo. Galaxy: kinematics and dynamics. Binaries, wide

\section{Introduction}

In recent times evidence has accumulated indicating that the galactic halo formed at least in part by accretion of material from smaller extragalactic systems. This view is in accordance with the hierarchical theory of structure formation in the Universe, in which the first objects to form are small galaxies, which then merge into the larger structures observed today. A consequence of this theory is that substructures should still be present within the old stars of the galactic halo and disk. Thus, the merger history of our galaxy should show up in the distribution of old stars, as coherent tidal streams, tails, moving clusters, etc. (Majewski 2004, 2005 and references therein).

But quite apart from the merger history of our galaxy, the stellar halo we now observe is also the result of the dynamical disruption of many globular clusters. Such disrupted clusters should also leave their signatures in the motions of the halo stars and should show up as dynamically coherent groupings in phase space. Poveda et al. (1992) searched for such groupings among the nearby halo stars of the first Schuster et al. catalogues (Schuster \& Nissen 1988, 1989a, 1989b, henceforth SN1) and they were able to identify seven moving groups, characterized by the similarity of their integrals of motion (the energy $E$ and the $z$-component of the angular momentum $h$ ) as well as by their similar metallicities $[\mathrm{Fe} / \mathrm{H}]$. In particular, the Kapteyn and the Groombridge 1830 moving groups were re-discovered.

On the other hand, there exists a small number of old and extremely wide binaries $(a>$ $10000 \mathrm{AU}$ ) that are very difficult to understand dynamically (Allen et al. 2000b, Carney et al. 1997). In Table 1 we provide a few examples. The first six rows list parameters 
Table 1. Properties of some extremely wide, old binaries

\begin{tabular}{lccccc}
\hline & $\begin{array}{c}\text { W 1828 } \\
(\text { HIP 15396) }\end{array}$ & $\begin{array}{c}\text { G 15-10 } \\
(\text { HIP 74199) }\end{array}$ & $\begin{array}{c}\text { G 40-14 } \\
(-)\end{array}$ & $\begin{array}{c}\text { LDS 519 } \\
(\text { HIP 74235) }\end{array}$ & $\begin{array}{c}\text { BD+80 245 } \\
(\text { HIP 40068) }\end{array}$ \\
\hline$E\left(100 \mathrm{~km}^{2} \mathrm{~s}^{-2}\right)$ & -1163.1 & -847.3 & -1153.7 & -524.6 & -878.3 \\
$h\left(10 \mathrm{kpc} \mathrm{km} \mathrm{s}^{-1}\right)$ & -143.1 & -41.8 & -69.6 & -238.1 & -118.5 \\
$R_{\min }(\mathrm{kpc})$ & 7.6 & 1.4 & 1.5 & 5.0 & 6.0 \\
$R_{\max }(\mathrm{kpc})$ & 9.6 & 30.2 & 15.1 & 69.2 & 25.8 \\
$\left|z_{\max }\right|(\mathrm{kpc})$ & 5.2 & 24.1 & 0.2 & 7.1 & 20.5 \\
Eccentricity & 0.12 & 0.91 & 0.81 & 0.87 & 0.62 \\
{$[\mathrm{Fe} / \mathrm{H}]$} & -2.05 & -2.42 & -2.46 & -1.28 & -2.07 \\
$s(\mathrm{AU})$ & 39590 & 89366 & 23030 & 8846 & 6513 \\
$\langle a\rangle(\mathrm{AU})$ & 55410 & 125073 & 32283 & 12380 & 9115 \\
\hline
\end{tabular}

of the galactic orbits of these binaries. The metallicity, $[\mathrm{Fe} / \mathrm{H}]$, is given in row 7 . The last two rows list the observed (projected) separations of the binaries and the expected major semiaxes, calculated according to the theoretical formula given by Couteau (1960). The values for the major semiaxes are quite large, and since these binaries are old, they should have been already dissociated. One possibility is that they have survived because they spend only a small fraction of their lives in the galactic disk, and they cross it at high speeds (Allen et al. 2000a). But perhaps there is another way for them to survive to the present day as binaries. They may, in fact, have been part of bound moving halo clusters, now dissolving or already dissolved.

The wide binaries and other substructures observed in the stellar halo of our galaxy may be the remains of either past mergers or disrupted clusters. An interesting question arises: how can we distinguish between both possibilities? Detailed chemical analyses can provide answers in some cases (King 1997, Ivans et al. 2003, Venn et al. 2004).

In the present paper we look for moving groups with the same technique used in Poveda et al. (1992), but we expand the SN1 sample with the more recent catalogue of Schuster et al. (2006), henceforth referred to as SN3. The total number of stars contained in the expanded sample is 1451 , out of which 483 turn out to be halo stars according to the $V_{\text {rot }}$ - $[\mathrm{Fe} / \mathrm{H}]$ criterion advocated by Schuster et al. to distinguish between halo, thick disk and disk stars.

The outline of this paper is as follows. In Section 2 we define our sample of stars and discuss the technique we used for identifying moving groups. Section 3 contains examples of possible halo moving groups, and compares these groupings with those of other workers who have used similar techniques. Section 4 explores the connection between our moving groups and some of the old and very wide binaries. Sections 5 and 6 study the connection between chemically peculiar stars and wide binaries or moving groups. Section 7 summarizes our main results.

\section{Identification of moving groups}

To identify moving groups we made use of the existence of two integrals of motion in an axisymmetric potential, namely the energy, $E$, and the $z$ component of the angular momentum, $h$. We computed these quantities for the halo stars of the combined sample SN3. We next looked for groupings in the $(E, h)$ plane, considering only stars having similar values of the metallicity $[\mathrm{Fe} / \mathrm{H}]$. We will refer to these groupings with similar $E$, $h$, and $[\mathrm{Fe} / \mathrm{H}]$ as "phase space clumps". We choose the $(E, h)$ plane as the most straightforward way of plotting quantities that are strictly conserved in a time-independent axisymmetric potential. Recent work (Pichardo et al. 2004, Allen et al. 2006) has shown that even in 

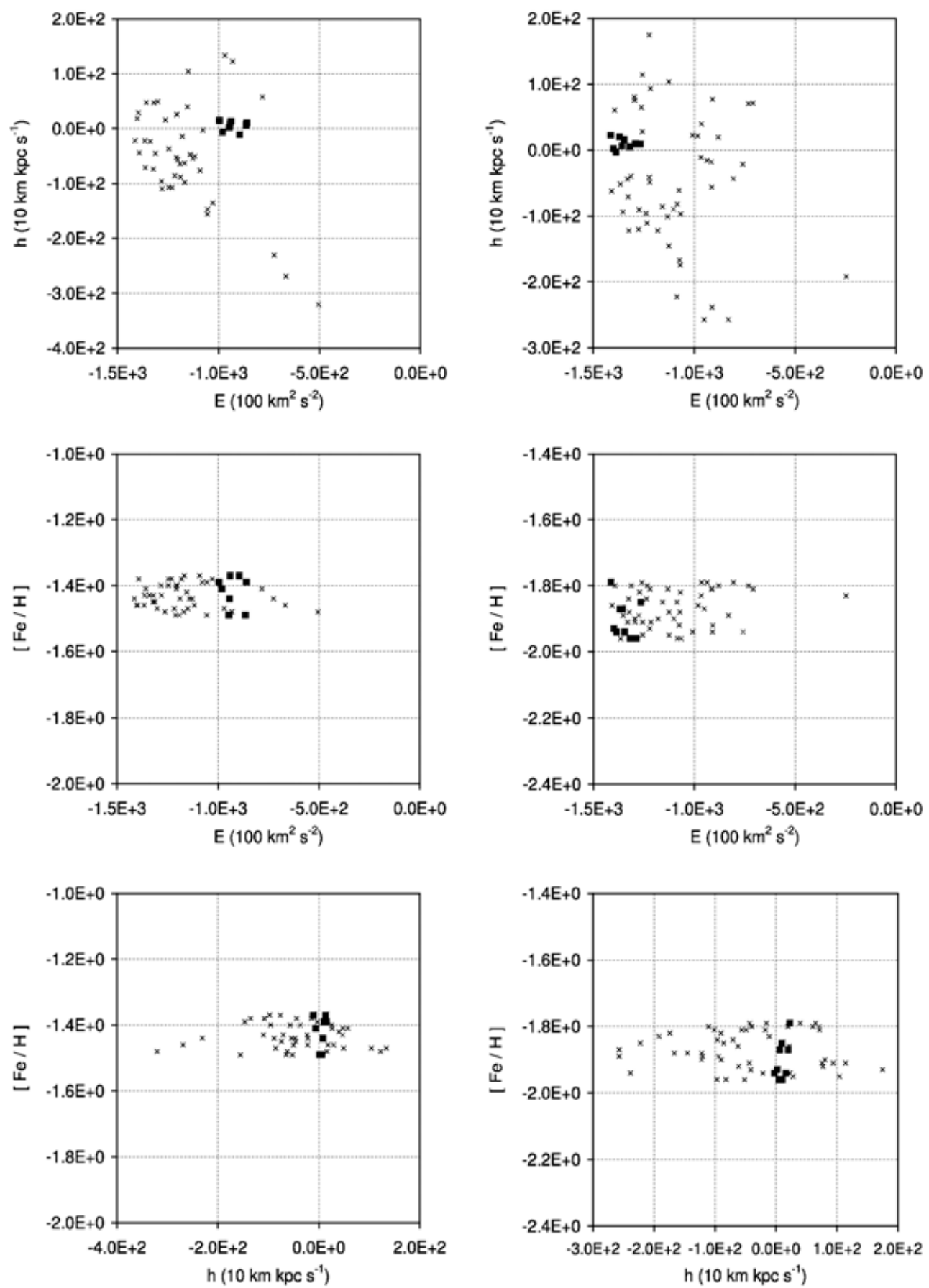

Figure 1. Left column. Plots of the two integrals of motion, $E$ and $h$, and of the metallicity, $[\mathrm{Fe} / \mathrm{H}]$ for the candidate moving cluster SN8. The stars belonging to this group are plotted as filled squares. The Schuster et al. (2006) stars with metallicities similar to those of the group members are plotted as crosses. Right column. Same plots for the candidate moving cluster SN11 
Table 2. Group 8 - members

\begin{tabular}{lccccccc}
\hline $\begin{array}{l}\text { Leading } \\
\text { star name }\end{array}$ & $\begin{array}{c}E \\
100 \mathrm{~km}^{2} \mathrm{~s}^{-2}\end{array}$ & $\begin{array}{c}h \\
10 \mathrm{~km} \mathrm{kpc} \mathrm{s}^{-1}\end{array}$ & $\begin{array}{r}{[\mathrm{Fe} / \mathrm{H}]} \\
\mid \begin{array}{c}z_{\max } \mid \\
\mathrm{kpc}\end{array}\end{array}$ & $\begin{array}{c}R_{\max } \\
\mathrm{kpc}\end{array}$ & $\begin{array}{c}R_{\min } \\
\mathrm{kpc}\end{array}$ & $e$ \\
\hline $\mathrm{G} \mathrm{192-043}$ & -895.8 & -10.8 & -1.37 & 17.4 & 27.8 & 0.16 & 0.99 \\
$\mathrm{G} 088-023$ & -995.4 & 14.7 & -1.39 & 17.8 & 21.8 & 0.25 & 0.98 \\
$\mathrm{G} 202-065$ & -879.9 & -6.4 & -1.41 & 13.7 & 22.8 & 0.09 & 0.99 \\
$\mathrm{G} 018-024$ & -946.4 & 2.0 & -1.49 & 22.9 & 23.1 & 1.4 & 0.89 \\
G 179-054 & -942.7 & 8.1 & -1.44 & 20.8 & 24.9 & 0.2 & 0.99 \\
G 215-047 & -939.6 & 13.5 & -1.37 & 18.2 & 24.9 & 0.2 & 0.98 \\
G 072-006 & -864.4 & 6.4 & -1.49 & 23.7 & 29.9 & 0.1 & 0.99 \\
G 126-063 & -860.0 & 10.1 & -1.39 & 23.1 & 30.1 & 0.2 & 0.99 \\
\hline
\end{tabular}

Table 3. Group 11 - members

\begin{tabular}{lccccccc}
\hline $\begin{array}{l}\text { Leading } \\
\text { star name }\end{array}$ & $\begin{array}{c}E \\
100 \mathrm{~km}^{2} \mathrm{~s}^{-2}\end{array}$ & $\begin{array}{c}h \\
10 \mathrm{~km} \mathrm{kpc} \mathrm{s}^{-1}\end{array}$ & $\begin{array}{r}\mathrm{Fe} / \mathrm{H}] \\
\mid \begin{array}{c}\left|z_{\max }\right| \\
\mathrm{kpc}\end{array}\end{array} \begin{array}{c}R_{\max } \\
\mathrm{kpc}\end{array}$ & $\begin{array}{c}R_{\min } \\
\mathrm{kpc}\end{array}$ & $e$ \\
\hline LP 720-028 & -1399.9 & 2.3 & -1.93 & 6.7 & 9.1 & 0.04 & 0.99 \\
HD 116064 & -1320.2 & 4.9 & -1.96 & 8.3 & 10.7 & 0.08 & 0.99 \\
LP 770-071 & -1359.0 & 6.5 & -1.87 & 7.4 & 9.9 & 0.11 & 0.98 \\
LHS 2969 & -1267.3 & 9.6 & -1.85 & 8.2 & 11.9 & 0.16 & 0.97 \\
G 029-071 & -1290.4 & 9.8 & -1.96 & 7.9 & 11.4 & 0.16 & 0.97 \\
LP 673-106 & -1347.1 & 15.9 & -1.94 & 7.4 & 8.9 & 0.83 & 0.83 \\
G 088-027 & -1370.0 & 20.3 & -1.87 & 6.3 & 9.6 & 0.38 & 0.92 \\
HD 160617 & -1387.8 & -2.9 & -1.94 & 7.4 & 9.3 & 0.05 & 0.99 \\
G 130-007 & -1414.0 & 22.4 & -1.79 & 5.4 & 8.8 & 0.41 & 0.91 \\
\hline
\end{tabular}

the presence of a bar, those quantities are also conserved on the average, except for orbits residing entirely within the bar, which is certainly not the case for relatively nearby stars such as the SN3 sample. Therefore, $E$ and $h$ will keep memory for long times of the values they had when they formed a group. We also require similar values of the metallicity, since the stars within a former group are likely to have but a small spread in their chemical compositions. A further requirement to identify group members is the similarity of their galactic orbital parameters, especially the maximum $|z|$-value.

As a criterion to define "similar values" we take the case of the well-studied common proper motion binary LDS 519 (= HIP 74234/74235 = HD 134439/134440). For this pair, we can compute the difference of the energies and $z$-components of the angular momenta of the galactic orbit of each component, as well as the difference in metallicity. Since there is little doubt that both components of this wide pair are physically connected, we can take these differences as representative of the differences we would expect to find among members of a common group.

\section{Halo moving groups among the SN3 stars}

We used the halo stars extracted from the Schuster et al. catalogues selecting them according to the population criterion $X$ advocated by these authors, which is a linear combination of the rotational velocity, $V_{\text {rot }}$, and the metallicity, $[\mathrm{Fe} / \mathrm{H}]$. A positive value of $X$ identifies a halo star.

In this way, we ended up with 478 stars belonging to the halo population. For these stars we computed galactic orbits using the Allen \& Santillán (1993) axisymmetric model potential. We looked for groupings in this halo star sample in the manner described above, 

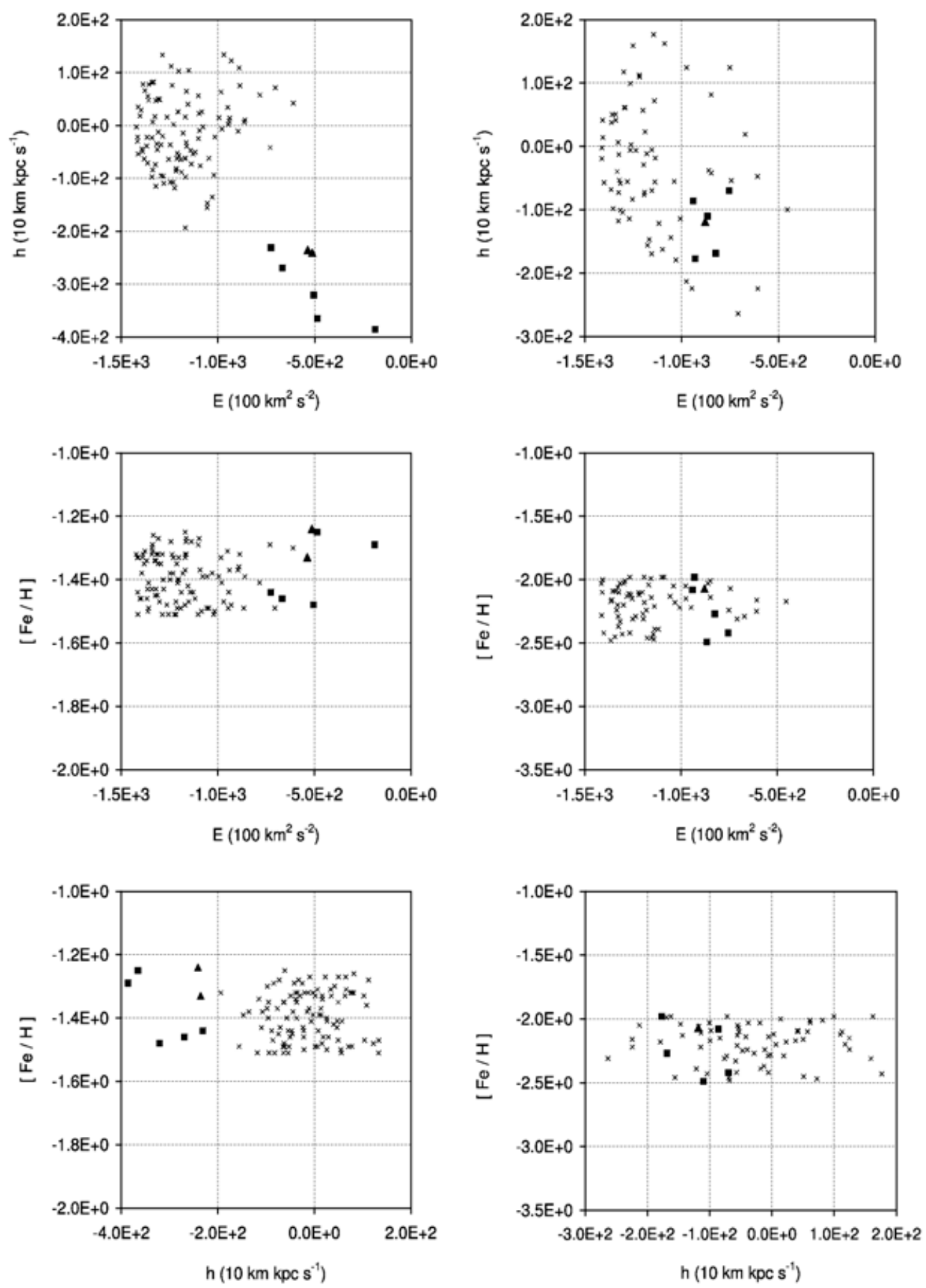

Figure 2. Left column. Plots of the two integrals of motion, $E$ and $h$, and of the metallicity, $[\mathrm{Fe} / \mathrm{H}]$ for the candidate moving cluster associated with the wide binary HD 134439/40. The stars belonging to this group are plotted as filled squares. The Schuster et al. (2006) stars with metallicities similar to those of the group members are plotted as crosses. Right column. Same plots for the candidate moving cluster associated with the wide binary BD $+80^{\circ} 245$. 
and we were able to identify about a dozen candidate moving clusters which satisfy the criteria of having similar values of $E, h,[\mathrm{Fe} / \mathrm{H}]$, and similar orbital parameters. A full discussion of these halo moving groups will be given elsewhere. Here, we only show a few examples. Tables 2 and 3 list the members of two candidate groups. Successive rows list the galactic orbital parameters. Figure 1 displays plots of their member stars in three planes containing combinations of $E, h$, and $[\mathrm{Fe} / \mathrm{H}]$. Note that the groupings are much tighter (especially in the planes containing $[\mathrm{Fe} / \mathrm{H}]$ ) than similar groupings proposed in the literature (Eggen 1996, Fiorentin et al. 2004, Chiba \& Beers 2000, Helmi \& White 1999).

\section{Wide binaries among moving groups}

Interestingly, we find wide binaries in 6 of our candidate moving clusters. These binaries were found by searching among the wide binaries of the catalog of Allen et al. (2000a), which was constructed using earlier versions of the Schuster et al. catalogues. The binaries were found independently of our search for moving clusters. The fact that some moving groups contain wide binaries supports our conjecture that some of the old wide binaries may be the survivors of former moving groups, which have already dissolved or are in the process of doing so. These binaries are listed in Table 4 . The rows and units in this table are the same as in Table 1.

Among the old wide binaries listed in Table 1 there are two well-studied objects that have very peculiar chemical abundances, namely LDS 519 and BD+80 245 (Chen \& Zhao 2005, King 1997, Zhang \& Zhao 2005, Ivans 2003, Zapatero-Osorio \& Martin 2004). Specifically, they show very low ratios of $[\alpha / \mathrm{Fe}]$ for their metallicities $[\mathrm{Fe} / \mathrm{H}]$. Such chemical peculiarities point to an enrichment history different from that of the bulk of our galactic halo. In fact, it has been suggested that they are reliable indicators of accreted structures. LDS 519 is contained in the SN3 catalog, and its galactic orbit was obtained with the parameters given in that source. For $\mathrm{BD}+80^{\circ} 245$, not contained in the SN3 catalog, we were able to obtain an orbit using data on its distance and kinematics from Hipparcos and Carney et al. (1997).

\section{Moving groups around chemically peculiar stars}

We may ask, conversely, whether there is any evidence of stars with values of $E, h$ and $[\mathrm{Fe} / \mathrm{H}]$ similar to those of these two chemically peculiar stars. We emphasize that they are both members of wide binaries. Our findings are displayed in Figure 2. We can see that there are indeed other stars with values of $E, h,[\mathrm{Fe} / \mathrm{H}]$ that are similar to those of LDS 519 and BD $+80^{\circ} 245$, although the groupings are not as tight as the ones we showed before. No detailed chemical abundances (i.e., $[\alpha / \mathrm{Fe}]$ ratios) are available for these candidate moving cluster members, but it would be very interesting if they should also turn out to be peculiar. This would confirm their membership to the halo moving clusters here proposed, and would constitute evidence for their origin by accretion.

\section{Binaries or moving cluster members with peculiar chemical composition}

Another question we can ask is whether there are other binaries or moving groups with peculiar values of $[\alpha / \mathrm{Fe}]$. To find an answer, we made use of the detailed study of Venn et al. (2004). We looked in their Table 2 for information on $[\alpha / \mathrm{Fe}]$ for all the wide binaries of our catalog (Allen et al. 2000a), as well as for the candidate members of moving 


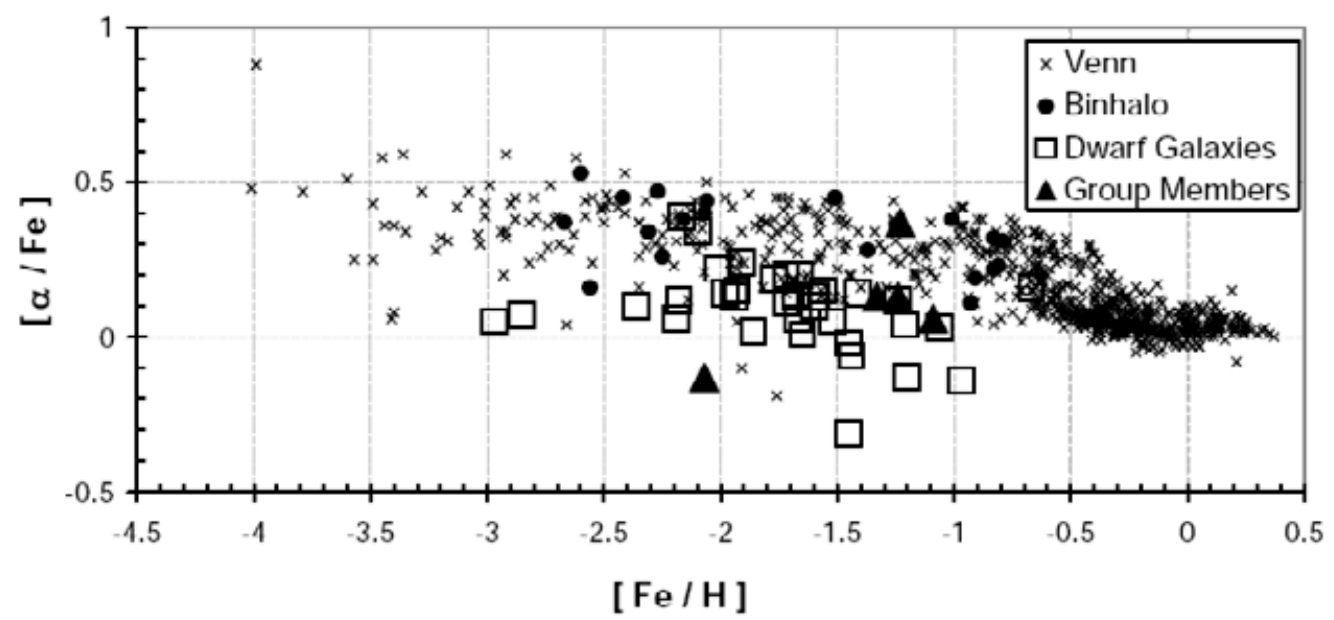

Figure 3. Plot of the abundance of $\alpha$ elements vs metallicity (adapted from Venn et al. 2004). The crosses denote stars, the empty squares, dwarf galaxies, as plotted by Venn et al.. We have added as dots the wide binaries of Allen et al. (2000a) for which $\alpha$-element abundances were available in Venn et al.. Triangles denote binaries that are members of moving clusters.

Table 4. Binaries in moving clusters

\begin{tabular}{lccccccc}
\hline & HD 105004 & HD 23439 & G 18-24 & G 40-14 & G 15-10 & HD 134439/40 & BD+80 245 \\
\hline$E$ & -1382.5 & -1304.3 & -946.4 & -1153.7 & -847.3 & -524.6 & -878.3 \\
$h$ & -19.8 & 99.4 & 2.0 & -69.6 & -41.8 & -238.1 & -118.5 \\
$R_{\min }$ & 0.3 & 3.1 & 1.4 & 1.5 & 1.4 & 5.0 & 6.0 \\
$R_{\max }$ & 9.3 & 9.6 & 23.1 & 15.1 & 30.2 & 69.2 & 25.8 \\
$\left|z_{\max }\right|$ & 6.1 & 1.5 & 23.0 & 0.2 & 24.1 & 7.1 & 20.5 \\
$e$ & 0.93 & 0.52 & 0.89 & 0.81 & 0.91 & 0.87 & 0.62 \\
{$[\mathrm{Fe} / \mathrm{H}]$} & -1.09 & -1.23 & 1.49 & -2.46 & -2.42 & -1.28 & -2.07 \\
$s$ & 1620 & 196 & 11782 & 23030 & 89366 & 8846 & 6513 \\
$\langle a\rangle$ & 2267 & 274 & 16489 & 32233 & 125073 & 12380 & 9115 \\
\hline
\end{tabular}

groups we identified among the SN3 stars. The result of this search is shown in Figure 3, adapted from Venn et al. (2004). We show as crosses the stars and as hollow squares the dwarf galaxies studied by Venn et al.. The wide binaries of Allen et al. appear plotted as thick dots, and the members of candidate moving groups as triangles. The figure shows that four of the stars identified as possible moving group members, namely HD 105004 , HD 134439/40 (LDS 519) and $\mathrm{BD}+80^{\circ} 245$ clearly have low $[\alpha / \mathrm{Fe}]$ for their $[\mathrm{Fe} / \mathrm{H}]$. Recall that $\mathrm{BD}+80^{\circ} 245$ is itself a wide binary, although only the primary is plotted in Figure 3. Such low $[\alpha / \mathrm{Fe}]$ stars are chemically similar to the dwarf galaxies, and are therefore probably accreted structures. Figure 3 also shows that at least five of our old wide binaries have such chemical peculiarities, and hence may also be the remains of accreted dwarf galaxies.

\section{Summary and conclusions}

Old wide binaries may have survived either because they spend little time in the galactic disk, crossing it at high speeds, or else because they were once members of bound clusters or still belong to moving groups. A search for "phase space clumps" among the stars of Schuster et al. (2006) yielded about a dozen groups of similar metallicities and 
galactic orbits. Previously known wide binaries turned out to be present in six of the identified moving groups. We may turn to detailed chemical composition analyses to distinguish whether a grouping had a galactic origin whether it is an accreted structure. Chemical peculiarities, like low $[\mathrm{O} / \mathrm{Fe}]$ or especially, low $[\alpha / \mathrm{Fe}]$ are characteristic of enrichment by type Ia supernovae, and thus of a chemical history different from that of our galaxy. Along with high eccentricities of the galactic orbits, or retrograde motions, these chemical peculiarities are thought to be signatures of accreted structures (dwarf galaxies). At least 5 of our old wide binaries have such peculiarities, and 3 are members of moving groups, which thus may be the remnants of dwarf galaxies accreted onto the galactic halo.

\section{Acknowledgements}

Our thanks are due to W.J. Schuster for fruitful discussions and to P. Ronquillo for her help in preparing the typescript. C.A. is grateful to the IAU for a travel grant.

\section{References}

Allen, C., Moreno, E., \& Pichardo, B. 2006, ApJ 652, 1150

Allen, C., Poveda, A., \& Herrera, M.A. 2000a, A $\& A$ 256, 529

Allen, C., Poveda A., \& Herrera, M.A. 2000b, in A. Weiss, T. Abel \& V. Hill (Eds.) The First Stars, Berlin: Springer, p. 66

Carney, B.W., Wright, J.S., Sneden, C., Laird, J.B., Aguilar, L.A., \& Latham, D.W. 1997, AJ 114,363

Chen, Y.Q. \& Zhao, G. 2006, MNRAS 370, 2091

Chiba, M. \& Beers, T.C. 2000, AJ 119, 2843

Couteau, P. 2000, J des Observateurs 43, No.3

Eggen, O.J. 1996, AJ 112, 2661

Fiorentin, P.R., Helmi, A., Lattanzi, M.G., \& Spagna, A. 2005, A $\& A$ 439, 551

Helmi, A. \& White, S.D.M. 1999, MNRAS, 307, 495

Ivans, I., Sneden, C., James, C.R., Preston, G.W., Fulbright, P., Hoflich, A., Carney, W., \& Wheeler, J. 2003, ApJ 592, 906

King, J.R. 1997, AJ 113, 2302

Majewski, S.R. 2004, Publ. Astr. Soc. Australia 21, 197

Majewski, S.R. 2005, ASP Conference Series vol. 338, p. 240

Pichardo, B., Martos, M., \& Moreno, E. 2004, ApJ 609, 144

Poveda, A., Allen, C., \& Schuster W. 1992, IAU Symp. 149: The Stellar Populations of Galaxies p. 471

Schuster, W.J. \& Nissen, P.E. 1988, A\&A 73, 225

Schuster, W.J. \& Nissen, P.E. 1989a, $A \& A A$ 221, 65

Schuster, W.J. \& Nissen, P.E. 1989b, A\&A 222, 69

Shuster, W.J., Moitinho, A., Marquez, A., Parrao, L., \& Covarrubias, E.B. 2006, A\&A 445, 939

Venn, K.A., Irwin, M., Shetrone, M.D., Tout, C.A., Hill, V., \& Tolstoy, E. 2004, AJ 128, 1177

Zapatero Osorio, M.R. \& Martin E.L. 2004, A\&SA 419, 167

Zhang, H.W. \& Zhao, G 2005, MNRAS 364, 712

\section{Discussion}

TERRY Oswalt: Do any of the groups and fragile binaries you find coincide with streams found in the "Spaghetti Project" and similar searches for galaxy accretion streams? The Sloan SEGUE project may be an excellent new source of similar groups. 
AlLEN: The "Spaghetti" project uses only proper motions (no radial velocities are available), so we cannot compute for them $(E, h)$. We found no CPM binaries in the structures of Chiba \& Beers and Fiorenhin et al., but this may be because their proper motions are too small to appear in NLTT (or rNLTT). 